\title{
Evaluation of Applicability of 1D-CNN and LSTM to Predict Horizontal Displacement of Retaining Wall According to Excavation Work
}

\author{
Seunghwan Seo, Moonkyung Chung* \\ Department of Geotechnical Engineering Research \\ Korea Institute of Civil Engineering and Building Technology, Goyang-si, Republic of Korea
}

\begin{abstract}
During excavation works in downtown, stability and safety considerations of such excavations and constructions are crucial for which continuous wall structures with varying structural components are commonly used. Most of the current models used for this purpose are often complex, where the accepted parameters do not have a clear physical meaning. Moreover, accurate ground movement forecasts are challenging due to nonlinear and inelastic soil behavior. Therefore, this study proposes a method to predict the lateral displacement of the braced wall at each stage of excavation by using all the basic information necessary for braced wall design, including ground information of the excavation site, support methods such as the type of brace, location and stiffness, information about the neighboring buildings, and the results of numerical analysis. One-dimensional convolutional neural network and long shortterm memory network are used for estimation and prediction to develop an optimal prediction model based on well-refined but limited data. The applicability of the braced wall was confirmed for safety management by predicting the horizontal displacement of the braced wall for each stage of excavation. The proposed model can be used to predict the stability of the horizontal wall for each excavation step and reduce accident risks, such as collapse of the retaining wall, which may occur during construction.
\end{abstract}

Keywords-Excavation; wall displacement; neural network; prediction wall deflection; CNN-LSTM

\section{INTRODUCTION}

Owing to the increase in high-rise buildings in urban areas, an increasing number of excavations are being planned. It is important to consider the potential serviceability issues caused by the construction of these structures. To maintain the stability of excavations, continuous wall structures with varying structural components are commonly used. These components can help prevent ground movements and reduce the impact on nearby structures. A reasonable estimate of the lateral wall deflection profiles caused by braced excavations is critical to ensure safe and economical construction. Therefore, measurements during construction are critical for determining the stability of the braced wall during excavation. In particular, wall displacement is the primary sign of problems with stability of the braced wall. To monitor this during construction, it is periodically measured using an inclinometer that can measure the lateral displacement of the braced wall, and thus the risk is determined. In addition, the inclinometer is the only method for measurement of the lateral displacement of the braced wall during excavation throughout the entire construction stage. Therefore, monitoring the lateral wall displacement through the inclinometer is indispensable; however, the measurement cost increases drastically if it is installed on all braced walls at an excavation site. Currently, engineers measure the lateral displacement of the braced wall using an inclinometer at a section that is representative of the entire structure. Therefore, there are still limits to management in sections other than the representative section, and accidents sometimes occur in these sections. As such, the retaining wall displacement for the unmeasured section can be estimated using numerical analysis or interpolation of the database. However, it is difficult to accurately predict ground movement because soil is a complex material and has inelastic behavior. Although various numerical models consider various features of soil, many of these models are often complex, and the accepted parameters do not have a clear physical meaning. Factors that affect the behavior of retaining walls at excavation sites are very diverse, such as the type of ground, the presence of adjacent buildings, and the support and wall construction methods. Based on empirical analysis of measured displacements in a large number of case histories, it is a proven method [1-4] to identify the main parameters affecting the deformation behavior during excavation works, as well as to examine general trends and patterns. This empirical design method is currently used a lot by engineers, but it is more inaccurate than a numerical model. However, it requires enormous computing resources to use a numerical model to predict the retaining wall. Therefore, an artificial intelligence (AI) based approach in geotechnical engineering is being used to analyze the complex behavior of underground structures.

An artificial neural network (ANN) was used in many research [5-16] to estimate the lateral wall displacement in excavation works. As some research trend, ANN was also used by Kung et al. [11] to calculate the deflection of diaphragm walls caused by excavation in clays. Chern et al. [12] used a back-propagation neural network (BPNN) model to forecast lateral wall displacement in top-down excavation. Random forest (RF) algorithm was utilized by Zhou et al. [13] to anticipate ground settlements caused by the building of a shield-driven tunnel. For the inverse analysis of soil and wall parameters in braced excavation, Zhang et al. [14] used multivariate adaptive regression splines (MARS). For the determination of Earth Pressure Balance (EPB) tunnel-related maximum surface settlement, Goh et al. [15] used the MARS

*Corresponding Author. 
model. Xie and Peng [16] tested the prediction power of Random Forest (RF) modeling for estimating tunnel Excavation Damaged Zones (EDZs). Despite the widespread application of supervised learning algorithms in geotechnical engineering, they have not been frequently applied for lateral wall displacement prediction in deep braced excavations considering the anisotropic shear strength.

As such, various artificial intelligence techniques have been utilized in relation to the stability of the retaining wall at the excavation site. However, the research so far has been limited to the study of the prediction of the maximum displacement of the retaining wall at the time when the excavation work is completed. In order to determine the stability during excavation work, it is important to manage the displacement of the retaining wall during construction, that is, according to the excavation stage. Most of the accidents related to excavation work occur during the excavation process, but no attempt has been made to predict the displacement of the retaining wall during excavation work. Therefore, predicting not only the maximum displacement after the excavation work is completed, but also the displacement of the retaining wall at each stage of excavation is considered to be helpful in reducing collapse accidents that occur in actual excavation work and evaluating the stability of the retaining wall.

This study attempted to predict the lateral displacement of the braced wall at each stage of excavation by using all the basic information necessary for braced wall design, including ground information of the excavation site, support methods such as the type of brace, location, and stiffness, information about the neighboring buildings, and the results of numerical analysis. Therefore, one-dimensional convolutional neural network (1D-CNN) and long short-term memory (LSTM) network were used, and the applicability of the braced wall was confirmed for safety management by predicting the horizontal displacement of the braced wall for each stage of excavation.

\section{PREDICTION MODEL AND CONSTRUCTION METHODOLGY}

\section{A. 1-D CNN}

Predictions based on existing time series data mainly use deep learning algorithms $[17,18]$. CNN (Convolutional Neural Network) is a deep learning algorithm and an effective neural network for identifying patterns in data because it specializes in processing array data. Therefore, $\mathrm{CNN}$ utilizes various filters that can be used as shared parameters; in the case of two dimensions, it efficiently extracts and learns features from adjacent images while maintaining the spatial information of the image. CNN, which mainly uses two-dimensional data, can be applied to data feature extraction and data prediction analysis by utilizing one-dimensional time series data [19-21]. CNN has the advantage of enabling easier training based on minimal parameters and preprocessing of data. The following equation (1) describes the output of a CNN corresponding to one-dimensional input data.

$s(t)=(x * w)(t)=\sum x(a) w(t-a)$ where $x$ is the input data, $w$ is the kernel map, and $s(t)$ is the feature map, which is the output layer. The CNN algorithm consists of four steps. In the first step, the kernel, which has a weighted function as the input data, traverses in a certain flow, and several convolution products are calculated in parallel. In the second step, the values computed in parallel go through the activation function, and the features of the input data are detected and output to the feature map. In the third step, the pooling function is used in the pooling layer to reduce the feature data detected in the feature map. As described above, the CNN algorithm extracts the features of the data through the iterations of the CNN and pooling layers. In the last step, for the dataset extracted from the $\mathrm{CNN}$ and pooling layers, the data constructed in an array are transformed into a column vector array through the fully connected layer, and the features of the data are classified. Fig. 1 shows the structure of the 1D-CNN algorithm.

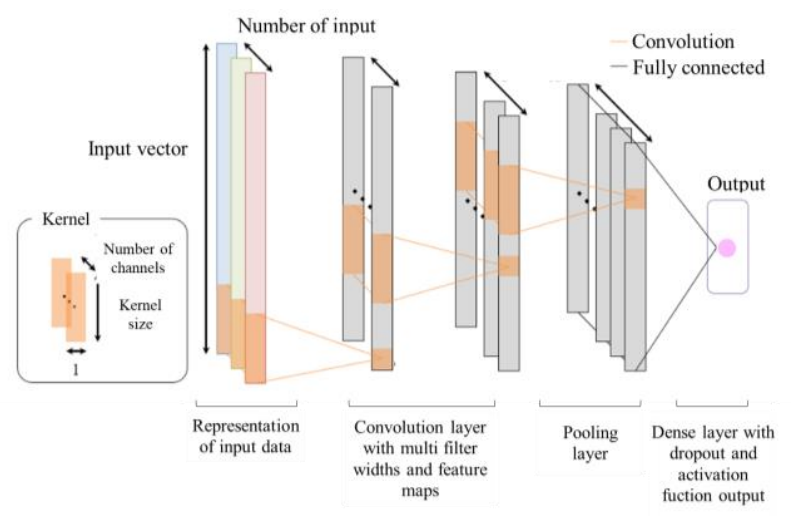

Fig. 1. Structure of 1-D CNN Algorithm.

\section{B. LSTM}

LSTM(Long-Short Term Memory) is mainly used for prediction and classification studies such as genes, handwriting, voice signals, sensor data, and stock prices [22]. Recently, many studies have been conducted to improve the prediction performance by modifying the structure of the LSTM [23, 24]. The LSTM algorithm was developed to solve the problem that owing to the structure of the recurrent neural network (RNN) algorithm, the time-series data of the distant past are not reflected if the data are large. The RNN algorithm transforms the hidden layer into forget, input gate, and output gates, which controls the flow of information to reflect timeseries data of the distant past. Fig. 2 shows the structure of the LSTM algorithm [25], in which X represents the input layer, $h$ represents the output layer, and a represents the hidden layer transformed into forget, input, and output gates.

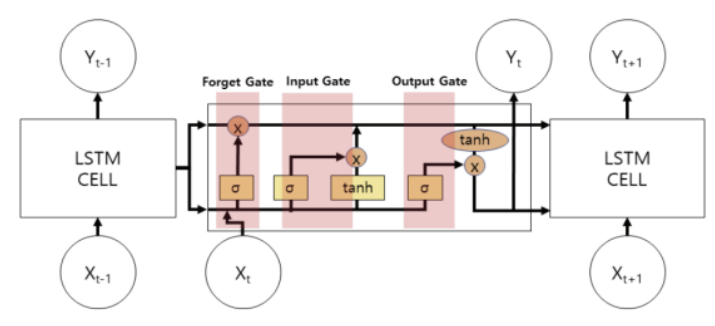

Fig. 2. Structure of LSTM. 


\section{Proposed Model}

The 1D CNN-LSTM model proposed in this study is a retaining wall displacement prediction algorithm to produce an optimal learning effect with limited iterative learning of timeseries data by combining CNN and LSTM (Fig. 3). The structure of the 1D CNN-LSTM model is divided into three stages. The first stage has a three-layer CNN structure and max pooling. In the first stage, the periodic and non-periodic features of the time-series data are extracted from the CNN layer, and a feature map is created using the output values. The max pooling layer is used to reduce the size of the extracted feature data. Max pooling selects the maximum value of the feature map. This process was repeated three times to extract the periodic and non-periodic features of the time-series data, and the data size was reduced significantly compared to the initial data size. The second stage consists of a flattened layer and a dense layer. The flattened layer converts multidimensional array data into 1D time-series data, and the dense layer connects both inputs and outputs. In the third stage, deep iterative learning of the LSTM layer was performed to ensure that the LSTM layer learns the relationship between the past and future data through the CNN. Future data were predicted based on the learned relationship.

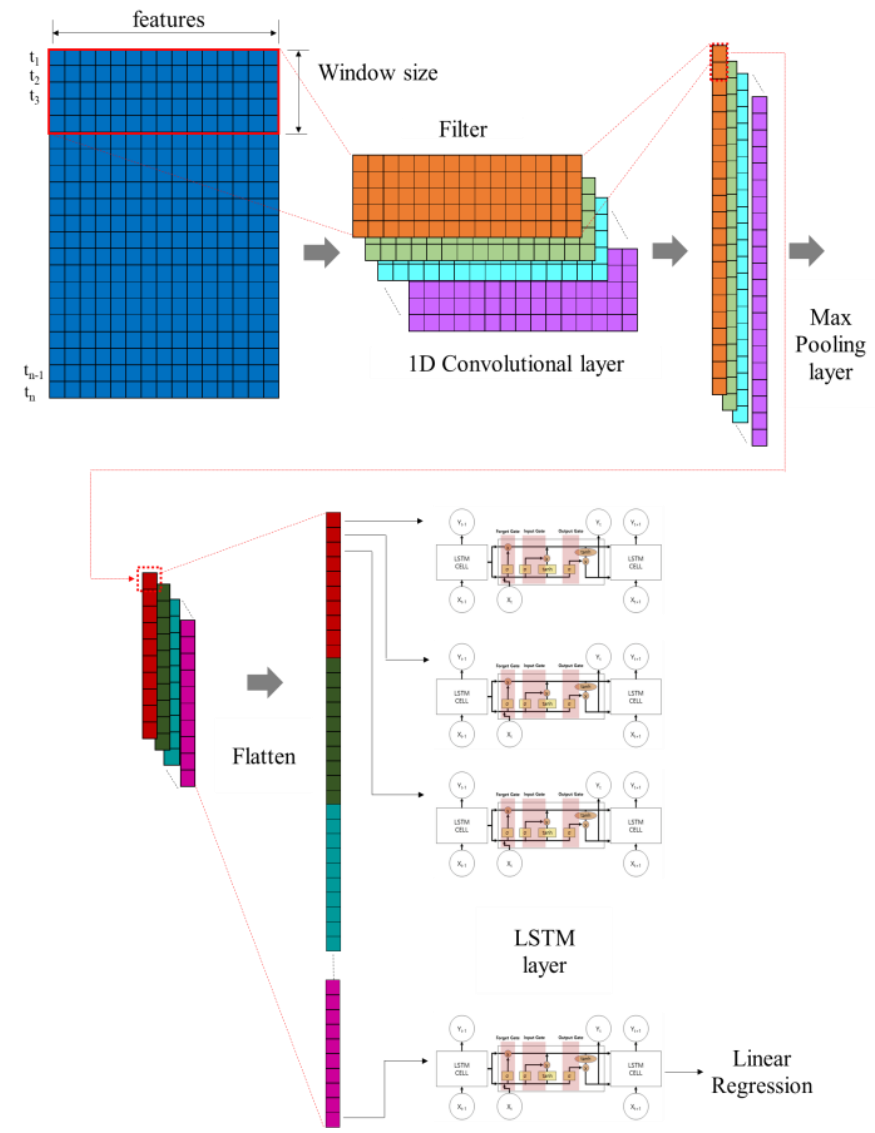

Fig. 3. Structure of Proposed Wall Deflection Prediction Model.

\section{Data Collection and Preparation}

Data collection is one of the most crucial steps in the prediction modeling. In this study, we need training data for the design and measurement over time to predict the horizontal displacement of the retaining wall for each excavation step. Therefore, we used the data obtained from excavation work sites in South Korea to prepare 30 input datasets by sorting the soil information, member information of the temporary retaining wall, numerical analysis results, and measurement results for each excavation step. The variables of the retaining wall data included all factors affecting the displacement of the retaining wall, such as the location, ground layer formation, soil strength, height of the retaining wall, height of the upper weak layer, retaining wall type, rigidity of the retaining wall, support type, and horizontal displacement of the ground. Fig. 4 is an example in which the various variables used as input data are scaled to a value between 0 and 1 and organized by depth. We could not collect a large amount of data because it was difficult to collect relevant information for step-by-step prediction from actual excavation sites. Therefore, the number of training data used in this study was relatively small, and we attempted to find the optimal model through cross-validation by changing the training and validation data.

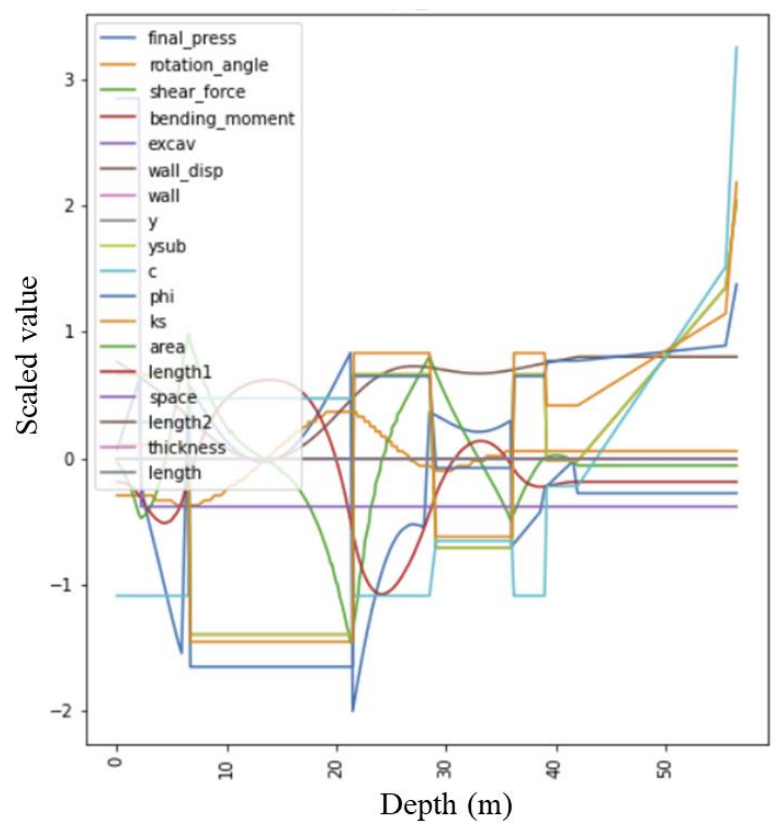

Fig. 4. Example of Preprocessed Input Data.

\section{RESULTS AND DISCUSSION}

Fig. 5 shows the overall accuracy and loss of the training and validation datasets. This shows that both the training loss and the validation loss start to converge above the 100th epoch. During this time, the overall accuracy of the training and validation tends to remain stable. Finally, the training was conducted for 1,000 epochs, and the optimal result was obtained at the 210th epoch. The performance improvement of the model cannot be expected through further training.

Because it was difficult to collect all excavation data for each step of the excavation work, the prediction values through cross-validation in this study were validated in this study. After training the model by excluding the design values of certain excavation site locations, the model by comparing the prediction values to the design values of those site locations were validated. 


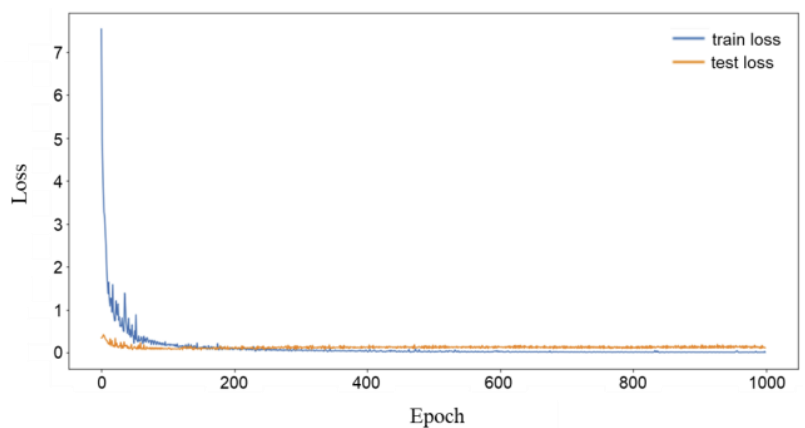

Fig. 5. Loss per Epoch during the Training of the Predicting Wall Deflection.

Fig. 6(a) and (b) show the predictions of the design values of excavation sites A and B, respectively, for each of the three excavation steps. The prediction results show that the changing trend of the horizontal displacement of the retaining wall is predicted properly in most cases. However, the horizontal displacement of the retaining wall was overestimated or underestimated at certain sites because the soil conditions and construction methods of the excavation sites were all different.

Fig. 6(a) shows that for site A, the horizontal displacement trend of the retaining wall is predicted well in every excavation step. Although there is a tendency to slightly overestimate the maximum horizontal displacement compared to the design value (true value), the depth at which the maximum horizontal displacement occurs matches exactly in each excavation step. This could help predict the position at which risk occurs before construction. Furthermore, the prediction values were mostly similar to the true values, regardless of the excavation depth in each excavation step.

In Fig. 6(b), site $B$ also shows that the horizontal displacement trend of the retaining wall is predicted well in every excavation step. Furthermore, the prediction value matched the true value for the depth at which the maximum horizontal displacement occurred. However, in contrast to site $\mathrm{A}$, the maximum horizontal displacement of the retaining wall was underestimated. In every case, it was determined that the accuracy of the prediction increases as the excavation progresses, and if the amount of training data increases, higher accuracy can be expected.

Fig. 7(a) and (b) show the predictions of the horizontal displacement of the retaining wall for certain cross-sections of sites A and B, respectively, for each excavation step. Here, the true value refers to the value measured using an inclinometer. For site A, it can be seen that the inclinometer measurement value and the prediction value match well in each excavation step. Furthermore, the predicted maximum horizontal displacement of the retaining wall is almost the same as the actual measurement value, and as the excavation progresses step-by-step, the difference from the actual measurement value decreases. For site B, few errors appeared to occur at low depths, but the trend of the displacement profile of the retaining wall was consistent. In actual measurements, the traffic on the surrounding roads and the adjacent buildings affect the ground. However, it is difficult to prepare these values in detail in the training data. Therefore, errors occurred at low depths close to the ground surface.
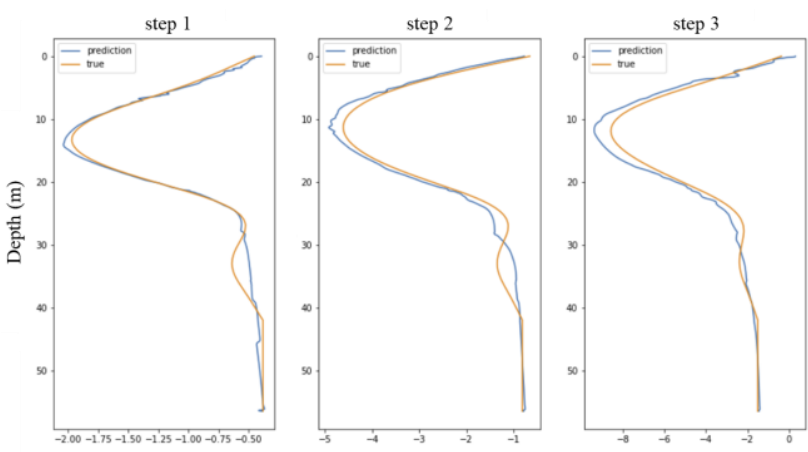

Wall deflection (mm)

Wall deflection $(\mathrm{mm})$

(a)
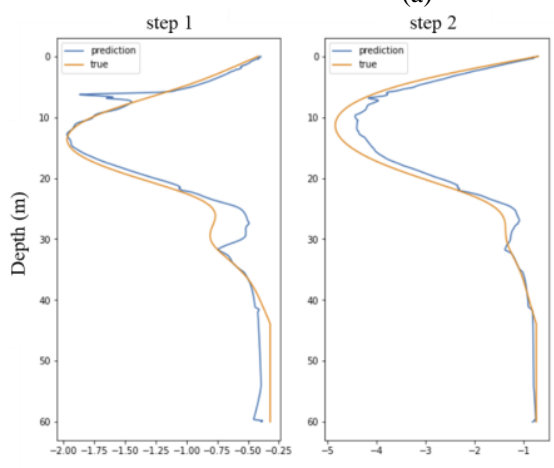

Wall deflection $(\mathrm{mm})$

(b)

Fig. 6. Wall Deflection Profiles with Numerical Analysis versus Prediction (a) Excavation Site A, (b) Excavation Site B.
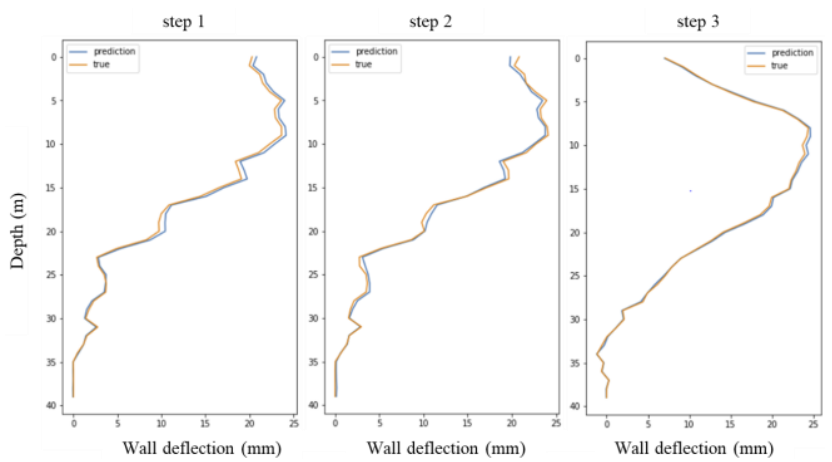

(a)
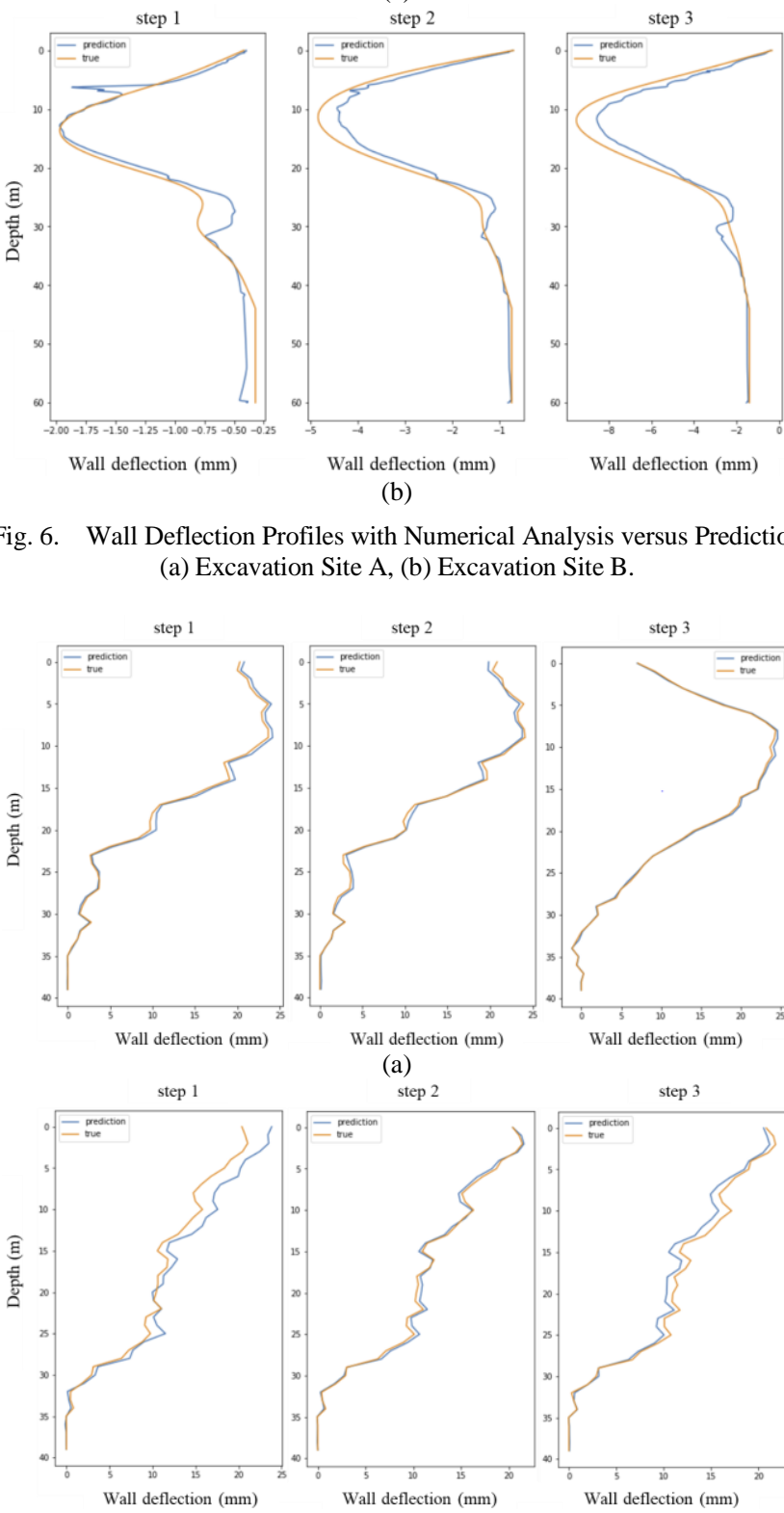

Wall deflection $(\mathrm{mm})$

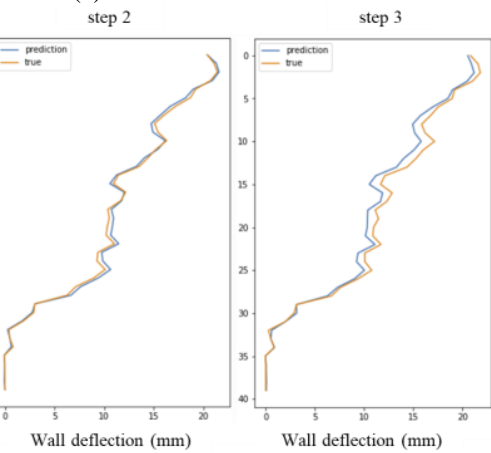

(b)

Fig. 7. Measured Wall Displacement Profiles versus Prediction (a) Excavation Site A, (b) Excavation Site B. 


\section{DISCUSSION}

In this study, a method combining CNN and LSTM was applied to predict the displacement of the retaining wall according to the excavation work step by step, and its applicability was confirmed. Through this study, it was found that the displacement change of retaining wall can be predicted as the excavation work progresses. While previous studies were limited to predicting the maximum displacement of the retaining wall, this study made it possible to measure the entire profile of the retaining wall. The ground inclinometer is the only measurement item that can reflect the entire excavation process, and the prediction accuracy can be improved by using this measurement value. In the previous study $[9,10,14]$ the maximum displacement prediction error of the retaining wall was about 6 23\%, but in this study, the prediction error for the maximum displacement was about $3 \sim 18 \%$. In addition, the prediction error for each stage of excavation was also confirmed to be about 15\%. Since the machine learning materials used so far may not be able to represent various environments such as all ground conditions and retaining wall construction methods, prediction errors may appear differently depending on the characteristics of the site. However, it is judged that the signal of accidents can be confirmed in advance by comparing the predicted results using the model proposed in this study with the retaining wall management standard according to the excavation work.

\section{CONCLUSION}

This study proposes a model that predicts the retaining wall displacement for each excavation step by combining 1D CNN and LSTM using the retaining wall measurement data. Compared to previous studies on the prediction of the maximum displacement of the retaining wall, this study has the advantage that the displacement profile of the retaining wall can be predicted for each excavation step. For highly reliable predictions, we need a large amount of data; however, we aimed to propose an optimal prediction model based on wellrefined data by training the model using limited training data and combining 1D CNN and LSTM. The results predicted by applying the measurement data of actual sites in the proposed model showed few differences from the actual measurement values. In these results, there was a tendency to show errors at certain sites because each site has different soil conditions and construction methods. Furthermore, this phenomenon seemed to be caused by the limited number of data, and this problem is expected to be solved by inputting additional measurement data in future.

For the safe management of the retaining wall during excavation work, predictions are required for not only the measurements of representative cross-sections but also for the unmeasured sections. In this regard, the proposed prediction model of this study can be used to predict the stability of the retaining wall for each excavation step and reduce accident risks, such as collapse of the retaining wall, which may occur during construction. Although the proposed model has some limitations, if appropriate data for the proposed model are collected and the database is built upon them, it could potentially help experts to use the model for designing or constructing retaining walls. Furthermore, it can help perform a more economical and safer retaining wall design or construction.

\section{ACKNOWLEDGMENT}

This research was supported by a grant from the project "Development of Smart Complex Solution for Large-Deep Underground Space Using Artificial Intelligence", which was funded by the Korea Institute of Civil Engineering and Building Technology(KICT).

\section{REFERENCES}

[1] Long M, "Database for retaining wall and ground movements due to deep excavations," J. Geotech. Geoenviron. Eng., 127, pp. 203-224, 2001.

[2] Moormann C, "Analysis of wall and ground movements due to deep excavations in soft soil based on a new worldwide database," Soils Found. Jpn. Geotech. Soc. 44(1), pp. 87-98, 2004.

[3] Wang ZW, Ng CWW, Liu GB, "Characteristics of wall deflections and ground surface settlements in Shanghai," Can. Geotech. J., 42, pp. 12431254, 2005.

[4] Ali J, Khan AQ, "Behaviour of anchored pile wall excavations in clays," Geotechn. Eng. 170(6), pp. 493-502, 2017.

[5] Goh, A.T.C., Wong, K.S., Broms, B.B., "Estimation of lateral wall movements in braced excavations using neural networks. Can. Geotech. J. 32 (6) (1995) 1059-1064.

[6] Hsiao, E.C.L., Kung, G.T.C., Juang, C.H., Schuster, M., Estimation of wall deflection in deep excavation - neural network approach," Geoshanghai Int. Conf., GSP, 155, pp. 348-354, 2006.

[7] Goh, A.T.C., Zhang, W.G., "An improvement to MLR model for predicting lique- faction-induced lateral spread using Multivariate Adaptive Regression Splines," Eng. Geol., 170, pp. 1-10, 2014.

[8] Adoko, A.C., Jiao, Y.Y., Wu, L., Wang, H., Wang, Z.H., "Predicting tunnel con- vergence using multivariate adaptive regression spline and artificial neural network," Tunn. Under. Space Technol. 38(3), pp. 368376, 2013.

[9] Zhang, W.G., Goh, A.T.C., Xuan, F., "A simple prediction model for wall deflection caused by braced excavation in clays," Comput. Geotech., 63 pp. 67-72, 2015.

[10] Zhang, W.G., Goh, A.T.C., "Multivariate adaptive regression splines and neural network models for prediction of pile drivability," Geosci. Front. 7, pp. 45-52, 2016.

[11] Kung, G.T.C., Hslao, E.C.L., Schuster, M., Juang, C.H., “A neural network approach to estimating deflection of diaphragm walls caused by excavation in clays," Comput. Geotech. 34 (5), pp. 385-396, 2007.

[12] Chern, S., Tsai, J.H., Chien, L.K., Huang, C.Y., "Predicting lateral wall deflection in top-down excavation by neural network," Int. J. Offshore Polar Eng., 19 (2), pp. 151-157, 2009.

[13] Zhou, J., Shi, X.Z., Du, K., Qiu, X.Y., Li, X.B., Mitri, H.S., "Feasibility of random- forest approach for prediction of ground settlements induced by the construction of a shield-driven tunnel," Int. J. GeoMech., 17 (6), $04016129,2017$.

[14] Zhang, W.G., Zhang, Y.M., Goh, A.T.C., "Multivariate adaptive regression splines for inverse analysis of soil and wall properties in braced excavation," Tunn. Undergr. Space Technol., 64, pp. 24-33, 2017.

[15] Goh, A.T.C., Zhang, W.G., Zhang, Y.M., Xiao, Y., Xiang, Y.Z., "Determination of earth pressure balance tunnel-related maximum surface settlement: a multivariate adaptive regression splines approach," Bull. Eng. Geol. Environ. 77, 489-500, 2017.

[16] Xie, Q., Peng, K., "Space-time distribution laws of tunnel excavation damaged Zones (EDZs) in deep mines and EDZ prediction modeling by random forest regression," Adv. Civ. Eng., pp. 1-13, 2019.

[17] Z. Chen, Y. Liu and S. Liu, "Mechanical state prediction based on LSTM neural network," in Proc. of the 2017 36th Chinese Control Conference (CCC), Dalian, China, pp. 3876-3881, 2018. 
[18] R. FukuoKa, H. Suzuki, T. Kitajima, A. Kuwahara and T. Yasuno, "Wind speed prediction model using LSTM and 1D-CNN," J-STAGE, 22(4), pp. 207-210, 2018.

[19] Y. H. Chen, T. Krishna, J. S. Emer and V. Sze, "Eyeriss: An energyefficient reconfigurable accelerator for deep convolutional neural networks," IEEE Journal of Solid-State Circuits, 52(1), 127-138, 2017

[20] F. J. Ordónez and D. Roggen, "Deep convolutional and LSTM recurrent neural networks for multimodal wearable activity recognition," Sensors. pp. 3-9, 2016.

[21] T. D. Do, M. T. Duong, Q. V Dang and M. H. Le, "Real-time selfdriving car navigation using deep neural network," in Proc. of the 2018 4th International Conference on Green Technology and Sustainable Development(GTSD), pp. 7-12, 2018.

[22] K. A. Althelaya, E. M. Alfy and S. Mohammed, "Evalutaion of bidirectional LSTM for short-and long-term stock market prediction," in
Proc. of the 2018 9th International Conference on Information and Communication Systems(ICICS), Irbid, Jordan, pp. 151-156, 2018.

[23] D. Niu, Z. Xia, Y. Liu, T. Cai and Y. Zhan, "Alstm: Adaptiv LSTM for durative Sequential data," in Proc of the 2018 30th International Conference on Tools with Artificial Intelligence (ICTAI), Volos, Greece, pp. 151-157, 2018.

[24] A. Graves, N. Jaitly and A. R. Mohamed, "Hybrid speech recognition with deep bidirectional LSTM," in Proc. of the 2013 IEEE Workshop on Automatic Speech Recognition and Understanding, Olomouc, Czech Repulic, pp. 273-278, 2013.

[25] Gers, F. A., N. N. Schraudolph, and J. Schmidhuber, Learning precise timing with LSTM recurrent networks. Journal of Machine Learning Research, pp. 115-143, 2002. 